Original Article

\title{
Analysis of scapular kinematics during active and passive arm elevation
}

\author{
Yoshiniro Kai, PT, $\mathrm{PhD}^{1)^{*}}$, Masafumi Gotoh, MD, PhD ${ }^{2)}$, Kazuto Takei, MD, $\mathrm{MS}^{3)}$, \\ Kazuya Madokoro, PT, MS ${ }^{4}$, Takeshi Imura, PT, PhD ${ }^{5}$, Shin Murata, PT, $\mathrm{PhD}^{1)}$, \\ Toru Morihara, MD, $\mathrm{PhD}^{6}$, , NaOto Shiba, $\mathrm{MD}, \mathrm{PhD}^{7)}$ \\ 1) Faculty of Health Science, Kyoto Tachibana University: 34 Yamada-cho, Yamashina-ku, Kyoto 607- \\ 8175, Japan \\ 2) Department of Orthopedic Surgery, Kurume University Medical Center, Japan \\ 3) Department of Physical Therapy, Technical School of Medical and Welfare, Japan \\ 4) Kurume University School of Medicine Graduate School, Japan \\ 5) Institute of Biomedical and Health Sciences, Hiroshima University, Japan \\ 6) Department of Orthopedics, Graduate School of Medical Science, Kyoto Prefectural University of \\ Medicine, Japan \\ 7) Department of Orthopedic Surgery, Kurume University, Japan
}

\begin{abstract}
Purpose] Early postoperative passive motion exercise after arthroscopic rotator cuff repair remains controversial. To better understand this issue, this study was aimed at evaluating scapular kinematics and muscle activities during passive arm elevation in healthy subjects. [Subjects and Methods] The dominant shoulders of 27 healthy subjects were examined. Electromagnetic sensors attached to the scapula, thorax, and humerus were used to determine three-dimensional scapular kinematics during active arm elevation with or without external loads and passive arm elevation. Simultaneously, the activities of seven shoulder muscles were recorded with surface and intramuscular fine-wire electrodes. [Results] Compared with active arm elevation, passive elevation between $30^{\circ}$ and $100^{\circ}$ significantly decreased the scapular upward rotation and increased the glenohumeral elevation angle. However, no significant differences in scapular posterior tilt and external rotation were observed between active and passive arm elevation, and scapular plane kinematics were not affected by muscle activity. [Conclusion] Unlike active motion with or without an external load, passive arm elevation significantly decreased the scapular upward rotation and significantly increased the mid-range glenohumeral elevation. These data, which suggest that passive arm elevation should be avoided during the early postoperative period, may expand the understanding of rehabilitation after arthroscopic rotator cuff repair.

Key words: Passive arm elevation, Scapular kinematics, Muscular activity
\end{abstract}

(This article was submitted Feb. 1, 2016, and was accepted Mar. 12, 2016)

\section{INTRODUCTION}

Rotator cuff tears are among the most common types of shoulder disorders. According to general population surveys, the prevalence of full-thickness rotator cuff tears is $20-25 \%$ among persons aged 50 years or older, and increases with age ${ }^{5)}$. Rotator cuff tears cause severe shoulder pain and restrict a person's range of motion, thus impeding activities of daily living.

Patients with rotator cuff tears are initially treated conservatively; however, surgical intervention is employed when the initial treatment fails. Postoperative physical therapy is also administered to allow the patients to regain their range of shoulder motion ${ }^{1-3)}$. In the study by Koo et al. ${ }^{4}$, it was suggested that early passive motion after arthroscopic rotator cuff

\footnotetext{
*Corresponding author. Yoshihiro Kai (E-mail: kai-y@tachibana-u.ac.jp)

(C)2016 The Society of Physical Therapy Science. Published by IPEC Inc.

This is an open-access article distributed under the terms of the Creative Commons Attribution Non-Commercial No Derivatives (by-nc-nd) License $<$ http://creativecommons.org/licenses/by-nc-nd/4.0/>.
} 
repair (ARCR) had beneficial effects on the range of motion and pain relief; similarly, in the report by Düzgün et al.6), it was demonstrated that an accelerated post-ARCR rehabilitation protocol prevented the negative effects of immobilization and supported rapid reintegration into activities of daily living. In contrast, in other studies, it was reported that early passive motion after ARCR may have a negative effect on cuff healing ${ }^{7)}$ and could not guarantee early gains in a patient's range of motion or pain relief ${ }^{8}$. Additionally, in the study by Cuff et al. ${ }^{9)}$, it was demonstrated that early aggressive rehabilitation protocols might yield a slightly higher incidence of re-tearing than do more conservative protocols. Therefore, the use of passive motion in the early postoperative phase remains controversial.

This controversy prompted the examination of scapular motion during passive arm elevation movements, frequently performed after ARCR, in this study. Accordingly, this study was aimed at determining the three-dimensional scapular kinematics and muscle activities around the shoulder during passive elevation with or without external loads. It was hypothesized that passive motion would alter scapular kinematics during arm elevation.

\section{SUBJECTS AND METHODS}

The dominant shoulders of 27 right-handed sedentary male subjects were examined. None of the subjects had any history of shoulder pain or injury before participating in this study. The subjects had a mean age of 22.5 years (range, 20-35 years), mean height of $173.3 \mathrm{~cm}$ (range, 160-185 cm), and mean weight of $66.1 \mathrm{~kg}$ (range, 55-85 kg). The study protocol was approved by the institutional review board of Kurume University (no. 09078), and informed consent was obtained from the subjects before their participation.

Three-dimensional thoracic, humeral, and scapular kinematic data were collected by using a LIBERTY electromagnetic tracking device (Pohlemus Inc., Colchester, VT, USA). This system comprised a low-frequency electromagnetic field transmitter, seven sensors (receivers), a stylus (digitizer), and a system unit. Data were collected at a sampling rate of $120 \mathrm{~Hz}$. The reported angular orientation accuracy was $1.3^{\circ 10}$, and the root mean square error due to skin motion artifacts was $<5^{\circ}$ at a humeral elevation of $<120^{\circ 11}$. Therefore, only data corresponding to shoulder elevation angles of $\leq 120^{\circ}$ were analyzed in this study. A global coordinate system was established by mounting the transmitter on a rigid wooden base frame and aligning it with the cardinal planes of the body. Electromagnetic sensors were attached to the sternum, superior acromion process, and humerus of the dominant arm. Bony thoracic, humeral, and scapular landmarks were palpated and digitized with the stylus sensor (digitizer) to establish an anatomically based local coordinate system (LCS). These procedures were performed while the subjects sat on a plastic chair with their arms relaxed at their sides. The LCS was chosen in accordance with the recommendations of the International Society of Biomechanics, as follows ${ }^{12)}$ : the $\mathrm{C} 7$ spinous process (C7), T8 spinous process (T8), incisura jugularis (IJ), and xiphoid process (PX) were used as thoracic landmarks; the glenohumeral rotation center (estimated through the rotation method), lateral epicondyle (EL), and medial epicondyle (EM) were used as humeral landmarks; and the acromial angle (AA), trigonum spinae (TS), and inferior angle (AI) were used as scapular landmarks. The thoracic superiorly directed axis (Yt-axis) extended from the midpoint between $\mathrm{T} 8$ and PX to the midpoint between $\mathrm{C} 7$ and IJ; the laterally directed axis (Zt-axis), which pointed rightward, was perpendicular to the plane defined by IJ, C7, and the midpoint between PX and T8; and the anteriorly directed axis (Xt-axis) was defined as the cross product of the Yt-axis and $\mathrm{Zt}$-axis. The humeral longitudinal axis (Yh-axis) extended from the midpoint between EL and EM to the glenohumeral rotation center; the anteriorly directed axis (Xh-axis) was perpendicular to the plane defined by the glenohumeral rotation center, EL, and EM; and the laterally directed axis (Zh-axis) was defined as the cross product of the Xh-axis and Yh-axis. The scapular laterally directed axis (Zs-axis) extended from TS to AA; the anteriorly directed axis (Xs-axis) was perpendicular to the plane defined by TS, AA, and AI; and the superiorly directed axis (Ys-axis) was defined as the cross product of the Xs-axis and Zs-axis.

Electromyographic (EMG) signals from seven shoulder muscles were recorded simultaneously by using a combination of surface and intramuscular fine-wire electrodes. EMGs were collected by means of 1,000-Hz sampling with a WEB-5000 multitelemeter system (Nihon Kohden Corp., Tokyo, Japan). This unit provides a signal amplification of 1,000×, common mode rejection ratio of $>54 \mathrm{~dB}$, input impedance of $>10 \mathrm{M} \Omega$, and gain of $100 \mathrm{~dB}$. The output from this unit was linked to a 16-bit analog-to-digital converter on a personal computer, and raw EMG and kinematic data from the electromagnetic sensor were synchronized and collected in MotionMonitor, version 8.43 (Innovative Sports Training Inc., Chicago, IL, USA) for offline analysis. Surface electrodes were used to record activity from the infraspinatus muscles, middle deltoid, serratus anterior, and upper and lower trapezius muscles at an interelectrode distance of $10 \mathrm{~mm}$. The electrodes were applied parallel to the muscle fibers after cleaning the skin with alcohol pads. The placement locations of the surface electrodes were selected according to published studies involving EMG data collection from the muscles of interest ${ }^{13}$ ). Bipolar intramuscular electrodes (50- $\mu \mathrm{m}$ urethane-coated stainless-steel wire; Unique Medical Co., Ltd., Tokyo, Japan) were used for the supraspinatus and subscapularis muscles. These intramuscular electrodes were prepared in accordance with the technique described by Basmajian and DeLuca ${ }^{14)}$ and were inserted according to the recommendations of Kelly et al. ${ }^{15}$ ) for the supraspinatus and those of Kadaba et al. ${ }^{16)}$ for the subscapularis after placement into 23-gauge single-use hypodermic needles. The signal quality was then checked to ensure adequate signal-to-noise ratios; poor-quality signals were excluded. The grounding electrode was affixed to the skin over the acromial end of the clavicle on the nondominant side. Correct electrode placement was confirmed by observing all EMG signals on an oscilloscope during resisted contractions of each muscle. EMG activities were recorded 
for all seven muscles while the subject performed maximum voluntary contractions (MVCs) against manual resistance, as previously described for shoulder normalization tests: abduction at $90^{\circ}$ with internal rotation ("empty can"), internal rotation at $90^{\circ}$ abduction ("internal rotation $90^{\circ}$ "), flexion at $125^{\circ}$ with scapular resistance ("flexion $125^{\circ}$ "), and horizontal adduction at $90^{\circ}$ flexion ("palm press") ${ }^{17)}$. Strong verbal encouragement was provided during every contraction to promote maximal efforts. EMG data from the MVCs were used to normalize the EMG amplitude (\%MVC) during the testing protocol.

Kinematic and EMG data were simultaneously collected during active and passive arm elevation in the scapular plane $\left(30^{\circ}\right.$ anterior to the coronal plane). Each subject was seated upright on a chair without a backrest and armrest. The arm was held at the side with the elbow extended $0^{\circ}$ in the starting position. Active arm elevation was performed under conditions of unloading or 3- and 5-kg loading. The subjects were instructed to raise their arms to full elevation in $3 \mathrm{~s}$ while a metronome was used for motion speed control. A posture hold bar and a cephalic strap were used to minimize motion during the experiment. Passive arm elevation was performed by using a motor-driven device (Fig. 1) that comprised a power unit and an arm elevation unit. The power unit was fitted with a 12-V direct current motor (DENSO Corp., Aichi, Japan) to drive the pulley. The arm elevation unit comprised a driven pulley and arm-bar connected to the power unit with a wire rope; this unit had a shaft output of $26.7 \mathrm{Nm}$ (actual measurement value) and a motion speed adjusted to reach full elevation in $3 \mathrm{~s}$. For passive elevation trials, the rotation center of the driven pulley was matched to the shoulder joint axis while the subject was in a sitting position. The forearm was fixed to the arm-bar with a cuff belt. Axial rotation of the humerus was not limited during arm elevation. Active and passive elevation motions were each performed two times, and the mean values of the two trials were analyzed. The subjects underwent sufficient practice before performing each elevation trial. Each subject randomly performed active and passive arm elevation to avoid testing bias. The repeat trial intraclass correlation coefficient (1.1) for the scapulothoracic and glenohumeral joint kinematic data ranged from 0.93 to 0.99 , indicating near-perfect reliability.

The scapulothoracic and glenohumeral joint angles were calculated at selected humeral elevation angles during the ascending phase by using MotionMonitor version 8.43 software. Humeral angles relative to the thorax were selected at $10^{\circ}$ intervals at arm elevations ranging from $20^{\circ}$ to $120^{\circ}$. Distal coordinate system rotations were described with respect to the proximal coordinate system, by using Euler angles in accordance with the recommendations of the International Shoulder Group of the International Society of Biomechanics ${ }^{12}$. The $Y X Z$ sequence was used to define scapular motions relative to the thorax. Rotational scapular motions were defined in the order of external/internal rotation (around the Ys-axis), upward/ downward rotation (around the Xs-axis), and anterior/posterior tilt (around the Zs-axis). Humeral motions relative to the scapula were determined by using the $Y^{\prime} X Y^{\prime}$ ' sequence, in which the first rotation defines the plane of elevation, the second defines the humeral elevation angle, and the third defines internal/external rotation. Humeral motions relative to the thorax were determined by using the $Y^{\prime} X Y^{\prime \prime}$ sequence as the elevation angle (second rotation). All kinematic data were smoothed with a Butterworth filter (low-pass) at a cutoff frequency of $8 \mathrm{~Hz}$. MotionMonitor was also used to synchronize the EMG and kinematic data. Raw EMG signals were band-pass filtered at 20-450 Hz, after which the root-mean square (RMS) amplitudes of the EMG signals were computed by using a 600-ms window. The median 100-ms RMS amplitudes at each humeral angle $\left(20-120^{\circ}\right)$ were used for the analysis. The RMS value of each muscle was then expressed as a percentage of the RMS value during the MVCs.

SPSS software for Windows version 22.0 (IBM, Armonk, NY, USA) was used for data analysis. The effects of load conditions (active or passive elevation) on the kinematic and EMG data collected during humeral elevation were analyzed by using a two-way repeated measures analysis of variance. When a significant main effect of the load condition was observed, the post hoc Bonferroni test was used to determine the significant differences between load conditions. The level of statistical significance was set at $\mathrm{p}<0.05$.

\section{RESULTS}

No significant interactions were observed between the load condition and arm elevation angle with respect to scapular internal rotation. Accordingly, neither active nor passive elevation influenced scapular internal rotation during arm elevation (Table 1). The load condition had a significant main effect $(\mathrm{p}<0.01)$ on the scapular upward rotation, and a significant interaction was observed between loading and the arm elevation angle $(\mathrm{p}<0.05)$. Accordingly, the scapular upward rotation was significantly lower with passive elevation than with active elevation, either with or without external loads, at arm elevations between $30^{\circ}$ and $100^{\circ}$ (Table 1). No significant interactions were observed between the load condition and arm elevation angle with respect to scapular posterior tilt. Therefore, neither active nor passive elevation influenced scapular posterior tilt during arm elevation (Table 1). The load condition had a significant main effect $(\mathrm{p}<0.01)$ on glenohumeral elevation, with a significant interaction between the loading and arm elevation $(\mathrm{p}<0.01)$. Accordingly, glenohumeral elevation was significantly greater with passive elevation than with active elevation at arm elevations between $40^{\circ}$ and $100^{\circ}$ (Table 1 ).

During arm elevation, the average muscle activities during passive elevation were consistently $<10 \%$ of the MVCs in all examined muscles. Muscle activities during arm elevation were significantly and primarily affected by the load condition $(p<0.01)$, with significant interactions between the load condition and arm elevation in all muscles except the supraspinatus $(p<0.01)$. Accordingly, all muscles exhibited significantly lower activity during passive elevation than during active elevation, regardless of the external load condition. The details are shown in Table 2. 

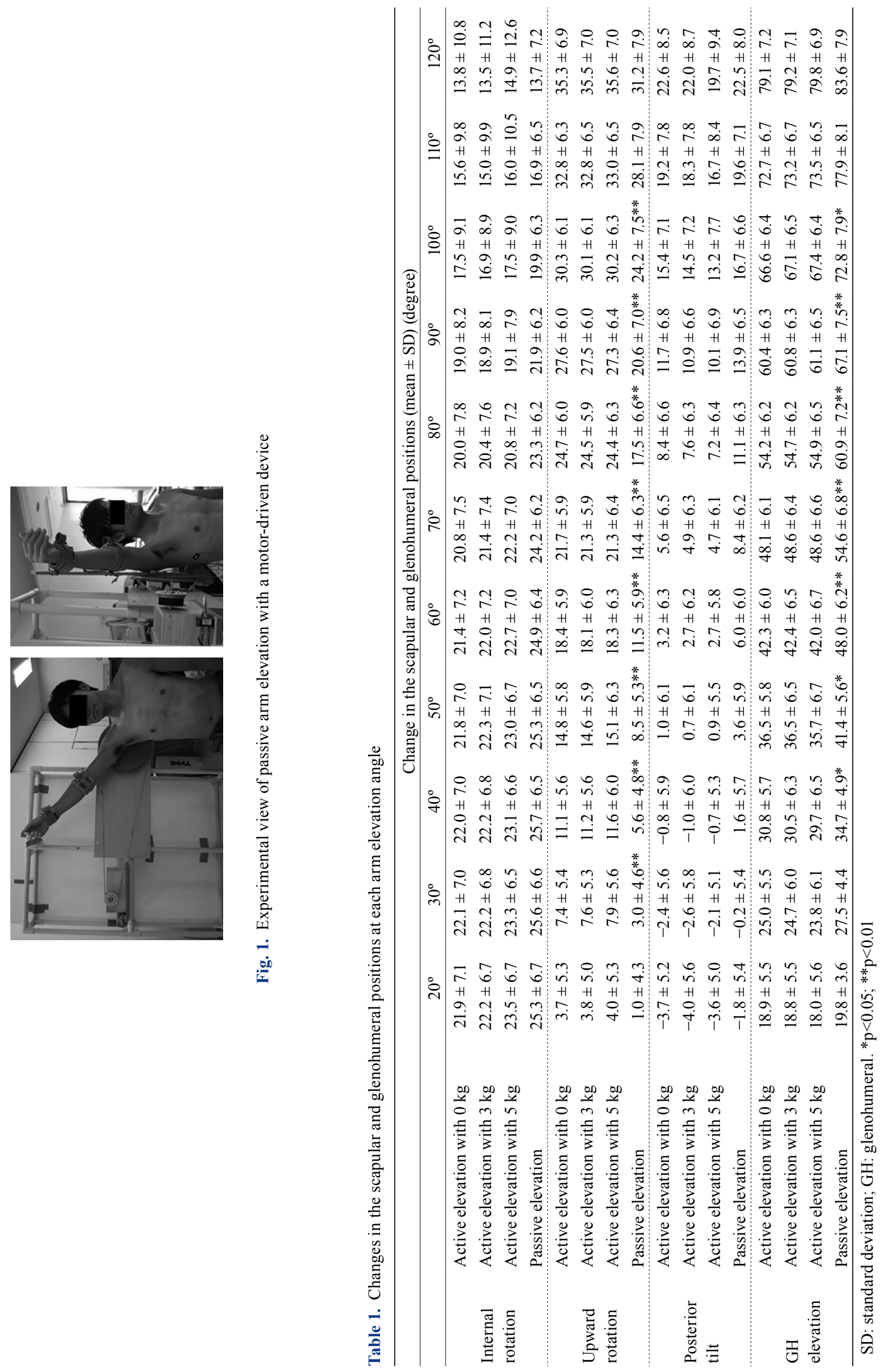


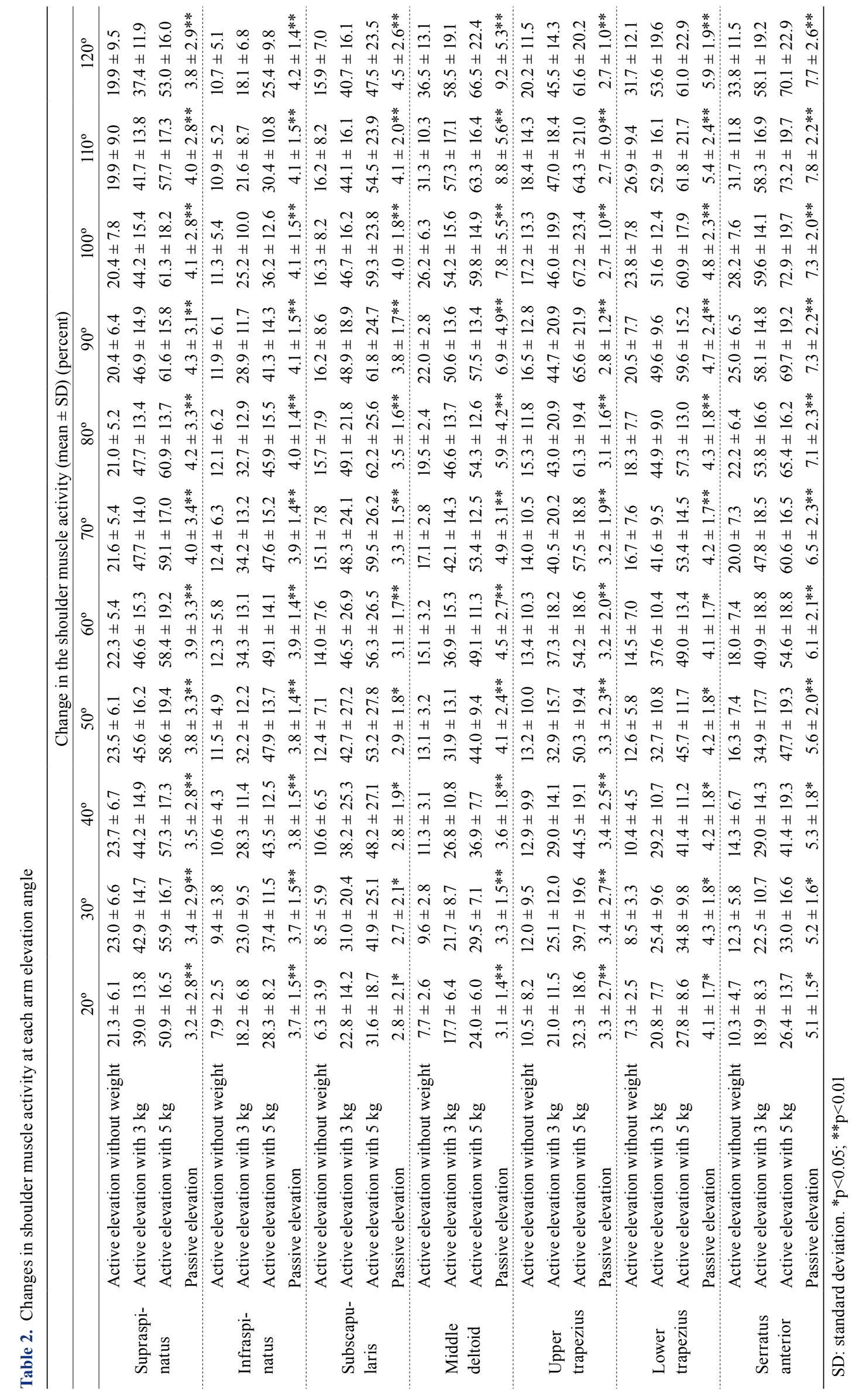




\section{DISCUSSION}

The focus of the present study was on the evaluation of scapular kinematics during passive arm elevation. The results indicated that passive elevation significantly reduced scapular upward rotation and increased glenohumeral motion at arm elevations between $30^{\circ}$ and $100^{\circ}$ versus active elevation with or without external loads; however, no significant differences in posterior tilt and external rotation were observed between passive and active elevation. Additionally, muscle activity increased parallel to the external load level, although the activity level did not alter the scapular kinematics. Therefore, unlike active elevation, passive elevation significantly reduced scapular upward rotation and increased glenohumeral motion in the mid-range of arm elevation.

Several studies have examined differences in scapular kinematics between active and passive arm elevation and have described a decrease in scapular upward rotation when the arm was passively elevated at $\geq 90^{\circ 18,19)}$. The data of the present study were consistent with those results; however, decreased upward rotation during arm elevation at angles $<90^{\circ}$. This difference appears to be associated with the passive elevation devices used in these studies, which were either a manual overhead pulley system ${ }^{18,19)}$ or an electrically controlled motor system in which the tested muscle activities were maintained at $\leq 10 \%$ of MCV.

Rotator cuff muscle activity reached a peak intensity during active elevation between $60^{\circ}$ and $90^{\circ}$. Previously, Saha ${ }^{20)}$ introduced a force-couple concept in which the subscapularis and infraspinatus co-stabilize the humeral head to the scapula during mid-range arm elevation. Barden et al. ${ }^{21)}$ demonstrated that the contributions of the supraspinatus and infraspinatus increase during mid-range elevation. Furthermore, Escamilla et al. ${ }^{22}$ ) demonstrated that the rotator cuff is activated during early humeral elevation. In the present study, decreased scapular upward rotation was observed during passive mid-range arm elevation, along with significantly decreased rotator cuff muscle activity. Therefore, the altered kinematics under conditions of passive elevation might affect the rotator cuff tendons (e.g., subacromial impingement ${ }^{23,24)}$ ).

In terms of the scapular posterior tilt and external rotation, no significant differences between active and passive arm elevation were observed at any humeral elevation angle. In previous studies, a lack of significant differences in the scapular posterior tilt and external rotation under these elevation conditions was observed ${ }^{18,19)}$. However, in the study by Thigpen et al. ${ }^{25)}$, scapular kinematic patterns were compared during internal and external humeral rotation at an elevation of $90^{\circ}$, and scapular posterior tilt was found to be associated with humeral rotation and the capsulo-ligamentous system. Therefore, the scapular posterior tilt and external rotation may not be influenced by active or passive motion during arm elevation.

In previous studies, the influence of external loading on scapular kinematics during arm elevation has been investigated $^{10,23,26)}$. In the report by McQuade and Smidt ${ }^{10)}$, heavy external loading was shown to cause an increase in scapular upward rotation during arm elevation. In contrast, in the study by Pascoal et al. ${ }^{26)}$, external loading did not influence scapular kinematics. Similarly, in the present study, scapular kinematics did not change in response to the external load.

In a recent systematic literature review, it was demonstrated that early passive rehabilitation protocols may result in a slightly higher incidence of re-tearing than that with more conservative protocols ${ }^{27}$. The data in this study indicated that passive motion resulted in a decreased scapular upward rotation and increased glenohumeral elevation during mid-range arm elevation. In the study by Muraki et al. ${ }^{28)}$, contact pressure in the subacromial space was examined during humeral elevation, and it was found that the highest contact pressure occurred at $62^{\circ}$, which corresponded to approximately $90^{\circ}$ of humeral elevation. Furthermore, in the report by Ludewig and $\mathrm{Cook}^{23)}$, it was demonstrated that scapular upward rotation was decreased at $60^{\circ}$ of humeral elevation in subjects with subacromial impingement. Taking these findings together, it can be suggested that early postoperative passive motion may increase the risk of re-tearing in the repaired cuff as a consequence of reduced scapular support; however, passive motion would be beneficial in cases of adhesive capsulitis because the glenohumeral motion is reduced ${ }^{29,30)}$.

The present study had several limitations. First, the analyzed range of humeral elevation was limited to $<120^{\circ}$ because of the requirements for accurate measurements using the electromagnetic sensors ${ }^{11}$. Second, the study subjects were healthy young adults, and it is unclear whether the results could be applied to patients with shoulder disorders. Third, the measurements were limited to only the scapular plane of elevation, and therefore it cannot be assumed that the results will be applicable to other planes of elevation (e.g., sagittal and coronal). Fourth, in the present study, power analysis was not performed before the experiment; therefore, there would have been a statistical error. Fifth, although the posture of the subjects were carefully checked and controlled before and during the experiment, there would have been an effect of sitting posture (increased thoracic kyphosis) on data analysis. Finally, the subacromial pressure associated with the decreased scapular upward rotation during passive arm elevation was not analyzed in this study. Resolution of these issues could lead to a better understanding of the influences of different loading conditions on scapular kinematics during arm elevation. 


\section{REFERENCES}

1) Goto M, Suzuki K, Shimada T: Relationship between lifestyle and shoulder motor function after reconstruction of rotator cuff tear. J Phys Ther Sci, 2007, 19: 243-249. [CrossRef]

2) Goto M, Yamamoto K, Morisaki Y, et al.: Effect of preoperative evaluation of rotator cuff injuries on the prediction of the convalescence period. J Phys Ther Sci, 2012, 24: 813-815. [CrossRef]

3) Matsuzaki T, Yoshida S, Kojima S, et al.: Influence of ROM exercise on the joint components during immobilization. J Phys Ther Sci, 2013 , 25: 1547-1551. [Medline] [CrossRef]

4) Koo SS, Parsley BK, Burkhart SS, et al.: Reduction of postoperative stiffness after arthroscopic rotator cuff repair: results of a customized physical therapy regimen based on risk factors for stiffness. Arthroscopy, 2011, 27: 155-160. [Medline] [CrossRef]

5) Minagawa H, Yamamoto N, Abe H, et al.: Prevalence of symptomatic and asymptomatic rotator cuff tears in the general population: from mass-screening in one village. J Orthop, 2013, 10: 8-12. [Medline] [CrossRef]

6) Düzgün I, Baltacı G, Atay OA: Comparison of slow and accelerated rehabilitation protocol after arthroscopic rotator cuff repair: pain and functional activity. Acta Orthop Traumatol Turc, 2011, 45: 23-33. [Medline] [CrossRef]

7) Lee BG, Cho NS, Rhee YG: Effect of two rehabilitation protocols on range of motion and healing rates after arthroscopic rotator cuff repair: aggressive versus limited early passive exercises. Arthroscopy, 2012, 28: 34-42. [Medline] [CrossRef]

8) Kim YS, Chung SW, Kim JY, et al.: Is early passive motion exercise necessary after arthroscopic rotator cuff repair? Am J Sports Med, 2012 , 40: 815-821. [Medline] [CrossRef]

9) Cuff DJ, Pupello DR: Prospective randomized study of arthroscopic rotator cuff repair using an early versus delayed postoperative physical therapy protocol. J Shoulder Elbow Surg, 2012, 21: 1450-1455. [Medline] [CrossRef]

10) McQuade KJ, Smidt GL: Dynamic scapulohumeral rhythm: the effects of external resistance during elevation of the arm in the scapular plane. J Orthop Sports Phys Ther, 1998, 27: 125-133. [Medline] [CrossRef]

11) Karduna AR, McClure PW, Michener LA, et al.: Dynamic measurements of three-dimensional scapular kinematics: a validation study. J Biomech Eng, 2001, 123: 184-190. [Medline] [CrossRef]

12) Wu G, van der Helm FC, Veeger HE, et al. International Society of Biomechanics: ISB recommendation on definitions of joint coordinate systems of various joints for the reporting of human joint motion-Part II: shoulder, elbow, wrist and hand. J Biomech, 2005, 38: 981-992. [Medline] [CrossRef]

13) Perotto A: Anatomical guide for the electromyographer: the limbs and trunk, 3rd ed. Springfield: Charles C. Thomas, 1994.

14) Basmajian JV, De Luca CJ: Muscles alive: their functions revealed by electromyography. Philadelphia: Lippincott Williams \& Wilkins, 1985.

15) Kelly BT, Cooper LW, Kirkendall DT, et al.: Technical considerations for electromyographic research on the shoulder. Clin Orthop Relat Res, 1997, (335): 140-151. [Medline]

16) Kadaba MP, Cole A, Wootten ME, et al.: Intramuscular wire electromyography of the subscapularis. J Orthop Res, 1992, 10: 394-397. [Medline] [CrossRef]

17) Boettcher CE, Ginn KA, Cathers I: Standard maximum isometric voluntary contraction tests for normalizing shoulder muscle EMG. J Orthop Res, 2008, 26: 1591-1597. [Medline] [CrossRef]

18) Ebaugh DD, McClure PW, Karduna AR: Three-dimensional scapulothoracic motion during active and passive arm elevation. Clin Biomech (Bristol, Avon), 2005, 20: 700-709. [Medline] [CrossRef]

19) Price CI, Franklin P, Rodgers H, et al.: Active and passive scapulohumeral movement in healthy persons: a comparison. Arch Phys Med Rehabil, 2000, 81: 28-31. [Medline] [CrossRef]

20) Saha AK: Dynamic stability of the glenohumeral joint. Acta Orthop Scand, 1971, 42: 491-505. [Medline] [CrossRef]

21) Barden JM, Balyk R, Raso VJ, et al.: Atypical shoulder muscle activation in multidirectional instability. Clin Neurophysiol, 2005, 116: 1846-1857. [Medline] [CrossRef]

22) Escamilla RF, Yamashiro K, Paulos L, et al.: Shoulder muscle activity and function in common shoulder rehabilitation exercises. Sports Med, 2009, 39: 663-685. [Medline] [CrossRef]

23) Ludewig PM, Cook TM: Alterations in shoulder kinematics and associated muscle activity in people with symptoms of shoulder impingement. Phys Ther, 2000, 80: 276-291. [Medline]

24) Bae YH, Lee GC, Shin WS, et al.: Effect of motor control and strengthening exercises on pain, function, strength and the range of motion of patients with shoulder impingement syndrome. J Phys Ther Sci, 2011, 23: 687-692. [CrossRef]

25) Thigpen CA, Padua DA, Morgan N, et al.: Scapular kinematics during supraspinatus rehabilitation exercise: a comparison of full-can versus empty-can techniques. Am J Sports Med, 2006, 34: 644-652. [Medline] [CrossRef]

26) Pascoal AG, van der Helm FF, Pezarat Correia P, et al.: Effects of different arm external loads on the scapulo-humeral rhythm. Clin Biomech (Bristol, Avon), 2000, 15: S21-S24. [Medline] [CrossRef]

27) Abtahi AM, Granger EK, Tashjian RZ: Factors affecting healing after arthroscopic rotator cuff repair. World J Orthod, 2015, 6: 211-220. [Medline] [CrossRef]

28) Muraki T, Yamamoto N, Zhao KD, et al.: Effects of posterior capsule tightness on subacromial contact behavior during shoulder motions. J Shoulder Elbow Surg, 2012, 21: 1160-1167. [Medline] [CrossRef]

29) Alptekin HK, Aydın T, İflazoğlu ES, et al.: Evaluatıng the effectiveness of frozen shoulder treatment on the right and left sides. J Phys Ther Sci, 2016, 28: 207-212. [Medline] [CrossRef]

30) Park SW, Lee HS, Kim JH: The effectiveness of intensive mobilization techniques combined with capsular distension for adhesive capsulitis of the shoulder. J Phys Ther Sci, 2014, 26: 1767-1770. [Medline] [CrossRef] 